\title{
LAADMINISTRACIÓN PÚBLICA Y SU INCIDENCIA EN LA POBREZA
}

\author{
Alexandra Herrera Zumba ${ }^{1}$, Roberto Vargas Zambrano ${ }^{2}$, Erick Ochoa Coronel ${ }^{3}$ y Jean Juca Alvarez . $^{4}$ \\ alexandra43@rocketmail.com¹,rovarza2005@yahoo.es², erickochoa2787@outlook.es³, \\ j juca29@ hotmail.com ${ }^{4}$ \\ https://orcid.org/0000-0002-9656-2336 ${ }^{1}$, https://orcid.org/0000-0001-8706-52932,

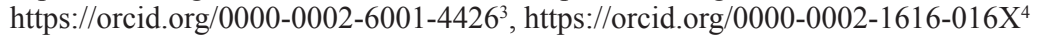 \\ Soluciones Integrales S.A. \\ Coronel Marcelino Maridueña - Ecuador
}

Recibido (07/08/20), Aceptado (21/08/20)

\begin{abstract}
Resumen: El Ecuador es un estado de gobierno republicano, democrático, intercultural, participativo y de administración descentralizada, según lo establecido en la Constitución de la República del Ecuador vigente desde el 2008. La administración pública de los gobiernos autónomos descentralizados municipales, es un principio de autonomía política previsto en la constitución y facultado por derecho en las normas y reglamentos de los poderes legislativo, ejecutivo y control ciudadano. La investigación es de tipo cuantitativa, teniendo como soporte la recolección y tabulación de la información obtenida de la muestra establecida a los jefes de hogares en el cantón Isidro Ayora del Ecuador, con el carácter de garantizar los derechos fundamentales como el buen vivir, siendo este el que agrupa todas las necesidades básicas que pueden ser: acceso libre al agua, alimentos, participación ciudadana, seguridad, hábitat, salud, educación, vivienda, trabajo, justicia, entre otros aspectos que benefician a la sociedad.
\end{abstract}

Palabras Clave: Pobreza, administración pública, política pública, gestión pública.

\section{PUBLIC ADMINISTRATION AND ITS IMPACT ON THE POVERTY OF A TOWN IN ECUADOR}

\begin{abstract}
Ecuador is a state with a republican, democratic, intercultural, participatory and decentralized administration, as established in the Constitution of the Republic of Ecuador in force since 2008. The public administration of decentralized municipal governments is a principle of political autonomy provided for in the constitution and empowered by right in the rules and regulations of the legislative, executive and citizen control powers. The research is quantitative in nature and is based on the collection and tabulation of information obtained from the sample of heads of household in the canton of Isidro Ayora in Ecuador, with the aim of guaranteeing fundamental rights such as the right to live well, which includes all basic needs such as free access to water, food, citizen participation, security, housing, health, education, housing, work and justice, among other aspects that benefit society.
\end{abstract}

Keywords: Poverty, public administration, public policy, public management. 


\section{I.INTRODUCCIÓN}

El Ecuador es un país en vías de desarrollo, cuyos niveles de pobreza son alarmantes en especial por la atención dada a los inexistente Objetivos del Milenio (ODM 2000 - 2015). En 2015, cerca de la décima parte de la población del mundo vivía en pobreza extrema, esta es la tasa más baja desde que se comenzó a llevar un registro de la pobreza. Este condicionante es notable, teniendo en cuente que en 1990 más de un tercio de la población mundial vivía en pobreza extrema [1].

La pobreza en el cantón Isidro Ayora del Ecuador, se puede evidenciar que el mayor índice de pobreza está en el sector rural, con un $80 \%$ entre sus 8.746 habitantes, siendo los factores de mayor incidencia la carencia de servicios básicos, tales como agua potable, alcantarillado, acceso a la educación y al servicio de salud, versus el $25 \%$ de pobreza entre sus 10.522 habitantes del sector urbano, como se demuestra entre los años 2018 al 2019 [2].

A pesar que los factores antes citados vinculados a la carencia de servicios básicos son determinantes, existen otras condiciones que históricamente tienen asidero en la pobreza y es de tan relevancia que predispone la actitud y decisión de ser o no ser, como lo es la cultura, tradición que heredan y deciden mantener, por ello el Banco Mundial en su Informe Anual del 2018 indica que prestan apoyo bilateral y asistencia a los países para hacer inversiones cruciales con el fin de generar un crecimiento inclusivo y sostenible de sus economías, crear el capital humano necesario para ayudar a las personas a aprovechar las oportunidades, garantizando que las naciones mantengan su resiliencia frente a las crisis y las amenazas mundiales que podrían socavar el avance de los esfuerzos encaminados a eliminar la pobreza [1].

El Instituto Costarricense de Pesca y Acuicultura (INCOPESCA) [3] realiza una precisión a lo que se refiere a las actividades económicas en su proyecto de desarrollo sostenible del sector pesquero y acuícola de Costa Rica, donde expone que se marcan diferencias en el sector de pesca de palangre, con el de pesca deportiva y turística, los pescadores artesanales de pequeña escala, quienes representan el mayor número, lo cuales se ubican en los cinturones de pobreza y pobreza extrema; contexto apropiado para adentrarnos en resolver los objetivos que se plantea en el proyecto investigativo como es evaluar mediante un estudio experimental los efectos que tiene el estudio sobre la pobreza en el cantón Isidro Ayora del Ecuador, generando condiciones, en que la reducción de la pobreza queda a merced de las políticas focalizadas de reducción de la pobreza, estas se deben ajustar al requisito de baja imposición, cuentas fiscales equilibradas y estabilidad macroeconómica, sa- crificando el crecimiento y el empleo.

Este trabajo se enfoca en la vinculación de la gestión, política y ética correspondientes a la administración pública y su efecto en las condiciones de vida, que desencadenan en la pobreza, y como la administración pública a través de sus estrategias podrían disminuir la pobreza en el cantón Isidro Ayora, provincia del Guayas, Ecuador.

En este trabajo podremos observar de manera resumida la investigación que se realizó en el cantón Isidro Ayora, provincia del Guayas, Ecuador. Donde se trabajó con 259 personas que viven en el cantón antes mencionados, los datos fueron obtenidos a través de un proceso de encuestas focalizadas en la administración pública y los condicionantes de pobrezas que existen, para su posterior interpretación y análisis estadístico de los datos extraídos de los ciudadanos del cantón Isidro Ayora del Ecuador, con la finalidad de emitir un análisis e interpretación de resultados a modo de discusión, donde se expondrá la realidad de la situación que vive el cantón versus un verdadero Buen Vivir. Para concluir con la propuesta, la misma que servirá de base para exponer nuestras conclusiones y recomendaciones en el desarrollo del presente trabajo.

\section{II.DESARROLLO}

El cantón Isidro Ayora cuenta con muchas etapas históricas, por ser una comunidad que fue habitada desde la época prehispánica, situación idónea para resaltar los hechos documentados que reposan en el gobierno local y que están a cargo de la biblioteca municipal y que hoy son expuestas al mundo para que sean acogidas por propios y extraños en sus proyectos investigativos, en este contexto es importante citar a la página Institucional del Gobierno Autónomo Descentralizado Municipal de Isidro Ayora y poder de esa forma conocer en breves rasgos la historia de Isidro Ayora.

En la época prehispánica, el actual territorio que ocupa la jurisdicción municipal del cantón Isidro Ayora, en la época prehispánica estuvo poblado como en la mayor parte de la provincia del Guayas, por tribus de Colonches, Chongones, Daulis y Mangachi, que, junto a los Ñausas, Oxivas, Palenques, Pimochas, Quilcas y Yaguachis, formaron parte de la etnia de los Huancavilcas, cuyo regulo era el cacique Guaya. El 2 de febrero de 1991 se constituyó un Comité Pro-Cantonización de Isidro Ayora, presidido por el profesor Andrés Indacochea Serrano, sra. Fabiola Jaramillo de Hinojosa (vicepresidenta) con un grupo de ayorenses comenzaron las gestiones de Cantonización más tarde la señora Fabiola Jaramillo de Hinojosa asume la presidencia del comité, el 2 y 3 de Julio de 1996 el Congreso Nacional Precedi- 
do por el Dr. Fabián Alarcón Rivera aprueba el proyecto de cantonización que fue elaborado y presentado por el Abogado Luis Almeida Moran; Diputado Provincial del Guayas.

El 2 de agosto de 1996 se publicó en el Registro Oficial \# 1002 de la Ley 134 sancionada por el Ministerio de la Ley del vigésimo octavo cantón del Guayas, en el gobierno del Arq. Sixto Durán Ballén [4].

\section{A.Antecedentes referenciales}

Comparando la información que proporcionó el censo de población y vivienda 2010 en lo que se refiere a pobreza por necesidades básicas insatisfechas, el cantón Isidro Ayora ocupaba el puesto trece entre los territorios más pobres del Ecuador, comparando con el estudio descrito en líneas precedentes, deja muy marcada la situación en que el gobierno desde sus distintos niveles ya sea central, provincial y local, no están realizando o articulando una correcta Administración Pública, con la finalidad de erradicar la pobreza, concordante con los inexistentes objetivos del Milenio ODM 2000 - 2015, transformados en los actuales Objetivos del Desarrollo Sostenible ODS 2015 - 2030, los estados miembros de la Organización de Naciones Unidas ONU se comprometieron en líneas generales poner fin a la pobreza, proteger el planeta y garantizar que todas las personas gocen de paz y prosperidad para 2030 [5], donde nos deja una interrogante específica y directa: ¿El discurso queda en papel, retorica o al fin se asume con claridad la responsabilidad de garantizarnos que la pobreza será erradicada?, a continuación se detalla los veinte cantones más pobres del Ecuador:

Tabla I. Pobreza por NBI - 20 cantones más pobres del Ecuador.

\begin{tabular}{cllccc} 
No. & \multicolumn{1}{c}{ CANTÓN } & \multicolumn{1}{c}{ PROVINCIA } & POBRES & TOTAL & POBRES \\
\hline $\mathbf{1}$ & TAISHA & MORONA SANTIAGO & 17.832 & 18.075 & $98,70 \%$ \\
$\mathbf{2}$ & MUISNE & ESMERALDAS & 27.942 & 28.433 & $98,30 \%$ \\
$\mathbf{3}$ & RIOVERDE & ESMERALDAS & 26.206 & 26.831 & $97,70 \%$ \\
$\mathbf{4}$ & OLMEDO & MANABI & 9.588 & 9.843 & $97,40 \%$ \\
$\mathbf{5}$ & MANGA DEL CURA & MANABI & 19.967 & 20.734 & $96,30 \%$ \\
$\mathbf{6}$ & TIWINTZA & MORONA SANTIAGO & 6.035 & 6.294 & $95,90 \%$ \\
$\mathbf{7}$ & ARAJUNO & PASTAZA & 6.208 & 6.486 & $95,70 \%$ \\
$\mathbf{8}$ & GUAMOTE & CHIMBORAZO & 43.079 & 45.098 & $95,50 \%$ \\
$\mathbf{9}$ & HUAMBOYA & MORONA SANTIAGO & 7.999 & 8.396 & $95,30 \%$ \\
$\mathbf{1 0}$ & 24 DE MAYO & MANABI & 27.338 & 28.797 & $94,90 \%$ \\
$\mathbf{1 1}$ & PAJAN & MANABI & 34.865 & 36.880 & $94,50 \%$ \\
$\mathbf{1 2}$ & ELOY ALFARO & ESMERALDAS & 37.391 & 39.569 & $94,50 \%$ \\
$\mathbf{1 3}$ & ISIDRO AYORA & GUAYAS & $\mathbf{1 0 . 2 5 3}$ & $\mathbf{1 0 . 8 5 2}$ & $\mathbf{9 4 , 5 0 \%}$ \\
$\mathbf{1 4}$ & CUYABENO & SUCUMBIOS & 6.157 & 6.518 & $94,50 \%$ \\
$\mathbf{1 5}$ & FLAVIO ALFARO & MANABI & 23.587 & 24.998 & $94,40 \%$ \\
$\mathbf{1 6}$ & LAS GOLONDRINAS & IMBABURA & 4.991 & 5.294 & $94,30 \%$ \\
$\mathbf{1 7}$ & URBINA JADO & GUAYAS & 54.017 & 57.311 & $94,30 \%$ \\
$\mathbf{1 8}$ & PEDRO CARBO & GUAYAS & 40.815 & 43.338 & $94,20 \%$ \\
$\mathbf{1 9}$ & SIGCHOS & COTOPAXI & 20.515 & 21.886 & $93,70 \%$ \\
$\mathbf{2 0}$ & PEDERNALES & MANABI & 51.442 & 54.899 & $93,70 \%$ \\
\hline
\end{tabular}

\section{B.Marco conceptual}

Jaramillo [6] expone que la administración pública es un proceso sistémico y metódico de carácter científico, técnico y jurídico. Acción que emprende los distintos niveles gobierno, en el cumplimiento de los objetivos institucionales de sus diversas entidades, con la finalidad de obtener los servicios previstos, planeados y programado sean atendidos de la mejor manera, con el mayor grado de eficiencia, eficacia y calidad para el desarrollo del país [6] [7].

En cambio, las políticas públicas permiten resolver un problema que tienen los ciudadanos de un determinado cantón [8], es el acto administrativo que ejecutan los distintos niveles de gobierno, en atender la agenda planteada por los sectores de la sociedad en su beneficio común, articulando los aspectos políticos, económicos, sociales, tecnológicos, ecológicos y legales [8] [9].

Por otra parte la gestión pública es la articulación que debe tener los distintos niveles de gobierno en eficien- cia, calidad, competitividad y eficacia de los sistemas de congruencia, financiero y operativo en la generación de tecnologías, procedimientos, bienes y servicios públicos [10] [11].

Se puede decir que la pobreza es una distopía en la que se encuentra la sociedad por la injusticia social de toda una vida, al no poder satisfacer las necesidades básicas humanas, convirtiéndose en carencias ilimitadas [12] [13] [14] [15].

\section{III.METODOLOGÍA}

En el proyecto de investigación se utilizó un enfoque mixto con la utilización de técnicas cualitativas y cuantitativas, que permitieron la cuantificación y tratamiento estadístico, además se empleó una metodología experimental, [16] la definen cuando el investigador manipula una o más variables de estudio, para controlar el aumento o disminución de esas variables y su efecto en las conductas observada. 
Siendo el investigador en plasmar el control de la variable independiente sobre de qué modo o por que causa efecto sobre otra variable; es importante considera que existen características muy marcados en esta investigación experimental, en este contexto se utilizó un diseño propiamente experimental solo de post-test con grupo control.

La metodología para los estudios políticos no se reduce a los métodos experimentales. También se cuenta con métodos cualitativos para hacer frente al dilema muchas variables, pocos casos y con métodos cuantitativos apoyados en instrumental estadístico y econométrico.

De acuerdo con los intereses del investigador, el análisis cualitativo puede ser simple o complejo, desde la descripción en lo micro hasta la explicación macropolítica pasando por la investigación cualitativa-comparativa y la causalidad que a su vez encuentra en la GT una herramienta para comprender las múltiples interacciones que se deben considerar [17].

De esta manera la investigación fusionó el análisis cuantitativo y cualitativo con la creatividad experimental. Habiendo evaluado el diseño de investigación, su tipo desde la metódica y adscripción epistemológica, nace la búsqueda en el sentido de su forma siendo esta netamente aplicada apoyada por investigaciones realizadas sobre las variables delimitadas en contexto nacional e internacional, que sirvieron para plasmado en lo local.

Su modalidad de investigación estuvo diseñada en el trabajo de campo, donde se formuló estrategias propias en la recolección de datos, siendo estos de gran ayuda el subcomponente virtual por ser relacionada en el campo académico, científico, laboral, personal entre otros.

Aplicando este muestreo de no probabilidades muestreo accidental determinamos que el tamaño de la muestra ajustada es de 259 jefes de hogares, siendo este el tamaño de la muestra definitiva que se requiere para nuestro proyecto investigativo en el cantón de Isidro Ayora, por su utilización en estimación de proporciones o resultados en porcentajes.

\section{IV.RESULTADOS}

\section{C.Satisfacción de servicios públicos en el cantón Isidro Ayora}

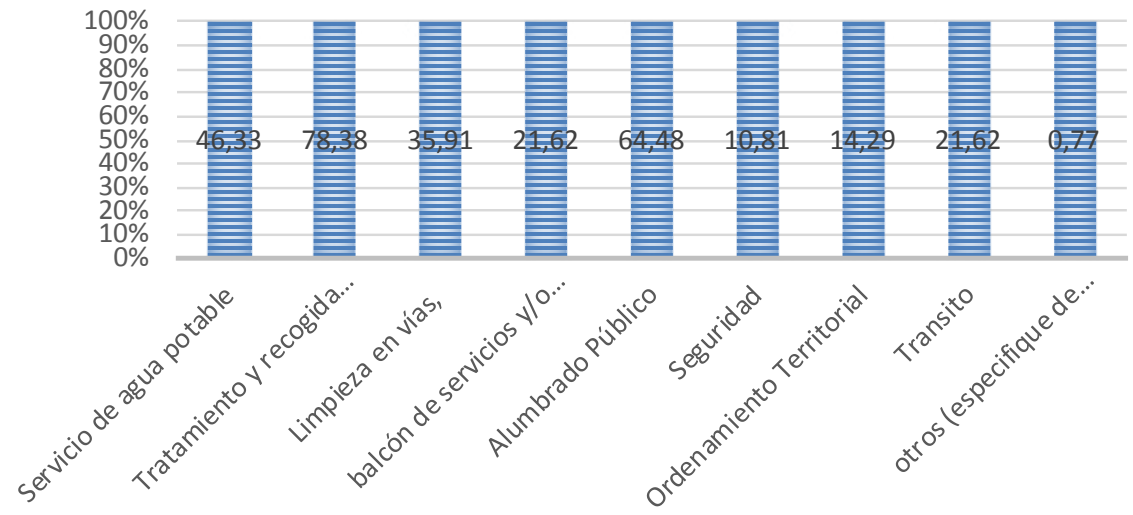

Fig. 1. Grado de Satisfacción de Servicios Públicos.

Muchas empresas públicas o privadas (agrupadas en un $44 \%$ ) ven en las encuestas, la forma más idónea de identificar el grado de satisfacción, por ello hemos enumerado una serie de servicios para que nuestros encuestados nos indiquen sus respuestas, en el marco de la SATISFACCIÓN, dando como resultado que el servicio de tratamiento y recogida de residuos sólidos (basura) goza del 78,38\% de aceptación, esto se origina porque el servicio en gran medida es puesto a disposición tanto a la zona urbana y rural.

Cabe indicar que nuestro cantón cuenta con 2 carros recolectores y al parecer la programación de sus recorridos es cumplido según lo planificado no dejando brechas de la desatención ciudadana, por otra parte el servicio de alumbrado público cuenta con el $64.48 \%$ de satisfacción, en este tema es notable indicar que en los lugares más lejanos ya cuenta con servicio y tendido eléctrico; lo alarmante de esta información es que los servicios de tránsito, seguridad y ordenamiento territorial ocupan los lugares más bajos, siendo novedoso agregar que existe un sector de entre los encuestados que proponen que el Servicio Turístico debe ser incluido en el análisis. 


\section{D.Evaluación de la gestión pública.}

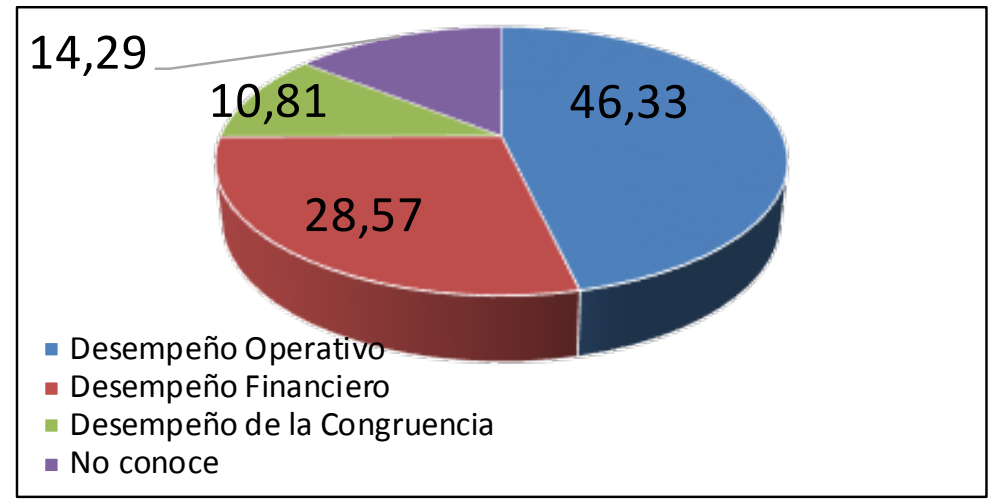

Fig. 2: Proceso de evaluación de la Gestión Pública.

La evaluación a la Gestión Pública es fundamental en la comprensión dinámica que se tiene frente a las necesidades básicas que tiene los habitantes de un sector determinado, ya que son herramientas en medir que tan eficiente son sus desempeños: operativo (administrativo y logístico), financiero (económico) y congruencia (interrelaciona de fuerzas sociales y técnicas) que tienen sus mandatarios en el desarrollo de sus actividades.

Para los encuestados poner mayor énfasis en el Desempeño Operativo 46,33\% significa que están evaluando a la Gestión Pública por lo tangible o lo que se denota, sin tomar en cuenta que deben ser una interrelación de los tres (3) tipos de desempeños operativo, financiero y de congruencia, ya que el mandatario debe ser integrador para poder tener todos los enfoques y poder de esta manera brindar alternativas de solución a los ciudadanos, esto es creando Políticas Públicas con programas, actividades y estrategias, focalizados en atender la pobreza vista de cualquier modelo o forma; también en la gráfica podemos apreciar que existe el $14.29 \%$ de la población que no conoce, preocupando porque es porcentaje alto de la población que no se preocupa o presta desinterés del desarrollo del ¿Qué hacer de la cosa pública?, que al final debe ser interés de todos y todas.

\section{E.Percepción del ciudadano en la dotación de servicios}

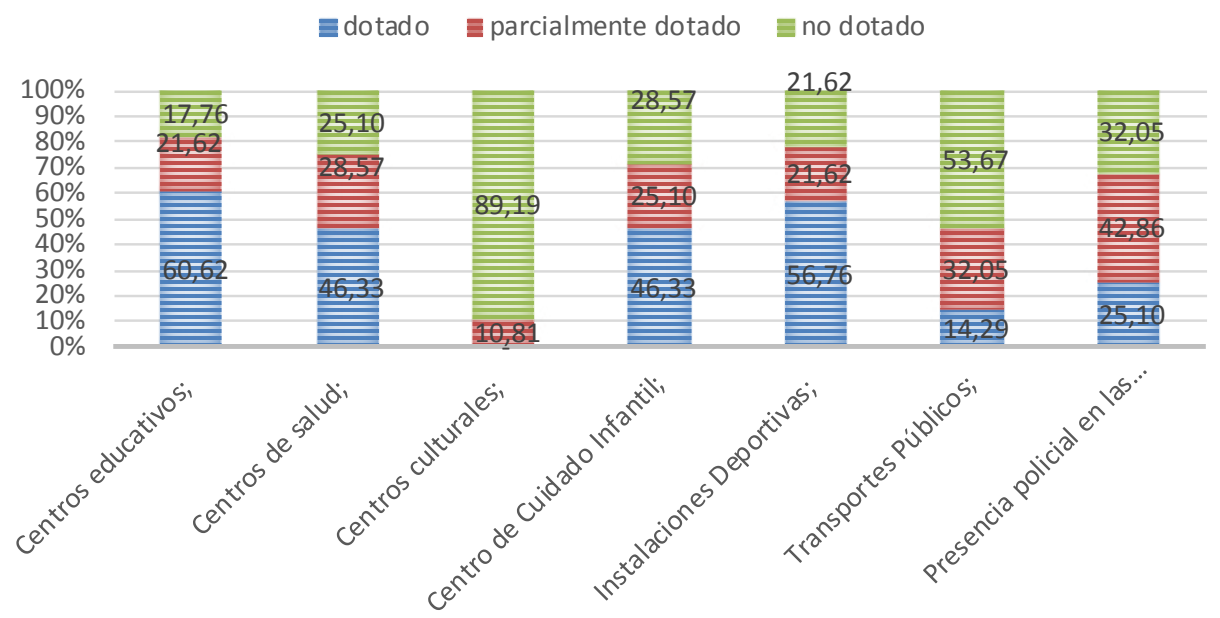

Fig. 3: Percepción del ciudadano en la dotación de Servicios Públicos.

Esta pregunta está dirigida en conocer como la Administración Pública debe aterrizar a las comunidades, además de poner la cara a los problemas que enfrenta la ciudadanía en general, en lo que respecta a centros culturales se expone una carente necesidad de dotar este tipo de infraestructura ya que no refleja información, de igual forma están los centros de salud que denota que existe una dotación del $46,33 \%$ cuando se conoce que el años pasado 26 abril de 2018 se inauguró el nuevo Centro de Salud Tipo A, con una atención exclusiva de 
solamente ocho (8) horas diarias de lunes a viernes, el transporte público es otro problema marcado en el cantón $53,67 \%$ no dotación, es un parámetro alto para las autoridades que busquen soluciones e intervengan en beneficios de todos.

Pero basta de datos deprimentes, tenemos otro espec- tro o aparentemente se las quiere pintar de esa forma, el cantón está dotado con el $60,62 \%$ de centros educativos, seguido por el $56,76 \%$ de instalaciones deportivas, esto quiere decir que, para nuestros encuestados, falta tener un Instituto de Educación Superior IES y un Complejo Deportivo de Alto Rendimiento.

\section{F.Percepción de la actuación del actual Alcalde del GAD Municipal de Isidro Ayora.}

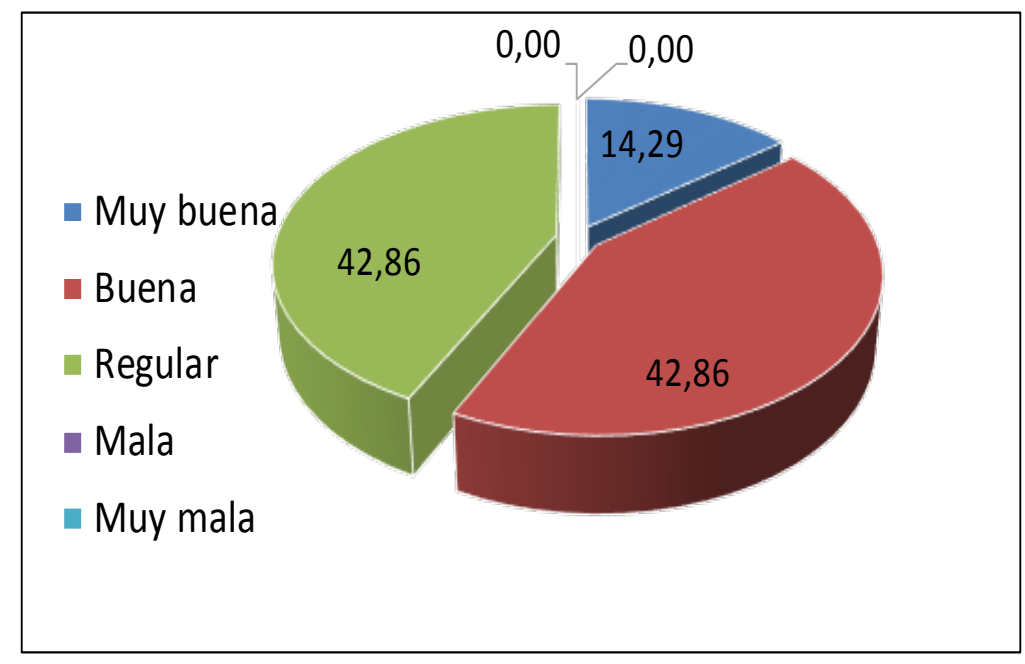

Fig. 4. Precepción de la actuación del actual Alcalde del GAD Municipal - Isidro Ayora.

En referencia a los resultados en calificar la actuación del actual burgo maestre, denotamos que no existen datos en las opciones de (mala y muy mala), pero se destaca que existe un $42,86 \%$ de encuestados que indican que es buena la administración, cabe indicar que ésta comienza en mayo/2019 apenas 4 meses, como dato adicional y pertinente se debe dar a conocer que el actual Alcalde, obtuvo una aceptación del $68.22 \%$ de sus electores.

En este contexto por ser una administración municipal que recién inicia sus actividades, los encuestados en gran mayoría expresan en los resultados una confianza plena a que sus aspiraciones sean cumplidas en el marco de una nuevo y reformado Concejo Cantonal, dicho en otras palabras, este Gobierno Municipal tiene la misión y visión de apuntalar sus acciones a que los habitantes puedan tener los servicios básicos y de esta manera satisfacer sus demandas ciudadanas.

\section{CONCLUSIONES}

La política social y de redistribución del ingreso son responsabilidades del gobierno y contribuyen a reducir la pobreza. Siendo los métodos más usados para medirla por el nivel de ingreso y las necesidades insatisfechas.

Los gobiernos locales deben contribuir a reducir la pobreza, desde las áreas de la salud, educación, vivien- da y alimentación, por el mismo sentido de ser el nexo directo e inmediato que tienen con las comunidades, cabe indicar que existen competencias exclusivas que tienen los distintos niveles de gobiernos, pero también es cierto que estos deben articular mesas y programas de cooperaciones interinstitucionales, donde además se vincule con el sector privado.

El gobierno central busca reducirla a través de programas sociales y programas asistenciales que de forma directa entregan bienes y servicios a la población de manera gratuita. En cambio, la política de redistribución del ingreso lo hace a través de subsidios o subvenciones, por ejemplo, el bono de la pobreza, precios de bienes y servicios subsidiados, etc.; estos programas sociales y las políticas de redistribución del ingreso contribuyen a aumentar el nivel de vida y reducir la pobreza.

En conjunto, la política social y de redistribución, posibilita mejores oportunidades de empleos e ingresos, siendo fundamental que las familias pobres obtengan ingresos de manera permanente, para generar empleo hay que incentivar el nivel de actividad económica, responsabilidad del sector privado y con apoyo del sector público.

En esta tarea de generar ingresos permanentes para los pobres, el gobierno local puede fomentar, promover 
e incentivar a sectores productivos, además de incentivar el turismo urbano y rural, impulsar la producción agrícola para la exportación ejemplo, productos orgánicos, productos con certificación de origen: mango, cacao, frejol, maíz entre otros.

En cuanto a salud, el gobierno local puede contribuir con la provisión de agua, recolección y procesamiento de basura (relleno sanitario), control de la contaminación de agua, aire y acústica (presentar un proyecto de ordenanza en que cobre una tasa por sus servicios). En las dos primeras contaminaciones es vital el control de agroquímicos en la agricultura, el control sobre todo en niños de bajo peso, anemia, baja estatura y derivar su atención al programa de salud pública.

Para la generación de empleo, el gobierno local, además de incentivar a sectores productivos puede realizar capacitación en emprendimientos para montar un negocio propio. El gobierno debe establecer el marco para el apoyo del emprendimiento (incubadoras de empresas, fondos para capital semilla y riesgo, líneas de crédito, etc.).

Es importante concluir que la pobreza para muchos suena como un mal necesario, pero para Isidro Ayora es una realidad que se vive y está muy lejos de acortar las brechas existentes, ya que no existe la más mínima intención de conocer sus niveles desde las autoridades de turno, en este sentido se puede concluir que los Gobiernos Autónomos Descentralizados, por el hecho de tener autonomía administrativa y financiera tiene el deber moral de ser los articuladores de la implementación de las Políticas Públicas como parte de la Administración Pública en dirigir acciones que fomenten erradicar la pobreza.

\section{G.Recomendaciones}

El presente trabajo de investigación por estar interrelacionado con todos los sectores, se inicia en recomendar a la comunidad en general del cantón Isidro Ayora, que sus limitados ingresos sean direccionados exclusivamente a satisfacer sus necesidades básicas.

De igual forma la Universidad ecuatoriana en su conjunto, debe tener la iniciativa de crear la Escuela de la Administración Pública, con el objetivo de preparar a la comunidad universitaria del cómo establecer mediante proyectos investigativos, soluciones a los problemas socioeconómicos de la sociedad en general.

Dando paso a que el Gobierno Autónomo Descentralizado Municipal de Isidro Ayora, tenga prioritariamente las siguientes iniciativas mediante Resolución del Concejo Cantonal:

1.Que la Jefatura de Gestión de Productividad, Eco- nómica, Social y Solidaria, lidere el proyecto de levantar un Sistema de Información Local periódica, donde se identifique o focalice territorialmente los niveles más altos de pobreza.

2.Que la Dirección de Gestión Financiera funcione como un verdadero soporte técnico en la elaboración de un verdadero Presupuesto Participativo, el mismo que deberá ser encaminado $u$ orientado a satisfacer bienes y servicios prioritarios en cumplimiento de sus competencias exclusivas.

3. Que el Concejo Cantonal haga funcionar sus competencias y que se establezca como un gobierno local vocero y coordinador ante los demás niveles de gobierno en monitorear el ciclo de las Políticas Públicas.

Siendo vista la pobreza desde el ingreso y necesidades básicas, es fundamental que el Gobierno Autónomo Descentralizado Provincial del Guayas, en cumplimiento de sus competencias intensifique la infraestructura vial para el desarrollo rural, ya que en la actualidad se presenta como un problema muy marcado a la hora de comercializar sus productos, siendo este sector desprotegido y por ende con los indicadores más altos de pobreza, acompañado en todo momento del Gobierno Central, el mismo que debe determinar la correcta agenda política, con la mayor intervención de los actores claves de la sociedad, para ello es importante conocer el problema el mismo que tiene la obligatoriedad de estar sustentado con bases de datos informativas confiables, para que en la ejecución de las políticas públicas estén dirigidas y focalizadas, con el soporte del análisis del entorno PESTEL.

En este contexto, no puede faltar el órgano asociativo por ello la Asociación de Municipalidades del Ecuador, tiene la necesidad de proponer como herramienta de trabajo el Modelo de Justicia Social, el mismo que deberá ser estratificado por necesidades básicas insatisfechas que presentan los grupos o categorías municipales, con el propósito de capacitar y dar el soporte técnico correspondiente con el fin de que no se desvíen, por lo contrario atiendan y cumplan los objetivos institucionales que deberán estar alineados con los objetivos del "Plan Nacional de Desarrollo 2017-2021 Toda una Vida" que impulsa el Gobierno Central.

El alcance internacional debe estar fusionado por ello el Centro Latinoamericano de Administración para el Desarrollo, debe tener el carácter de convocante a la comunidad académica y demás sectores de la sociedad en coordinación con la Administración Púbica, seamos parte de este organismo con la importancia de tener la oportunidad de presentar propuestas o alternativas de solución en la erradicación de la pobreza latinoamericana; que en coordinación con la Organización de Nacio- 
nes Unidas, formulara o impulsará un informe, donde no se mescle los llamados tipos de pobreza, con el fin de preparar una propuesta donde se obtenga una sola forma de medición y poder de esta manera conocer un verdadero índice de pobreza humana, que permita conocer el nivel de cumplimiento que tiene cada país miembro, frente a los Objetivos de Desarrollo Sostenible de la agenda 2030.

\section{REFERENCIAS}

[1]Banco Mundial, INFORME ANUAL 2018 - Poner fin a la pobreza. Invertir para generar oportunidades, Washington: Banco Mundial, 2018, p. 90.

[2]Gobierno Autónomo Descentralizado Municipal de Isidro Ayora, «PLAN DE DESARROLLO Y ORDENAMIENTO TERRITORIAL,» 15 Abril 2016. [En línea]. Available: http://app.sni.gob.ec/sni-link/ sni/PORTAL_SNI/data_sigad_plus/sigadplusdiagnostico/0960006850001_GAD\%20ISIDRO\%20 AYORA\%20PDYOT\%20ACT\%202014-2019_15-042016_05-21-05.pdf.

[3]P. Andrade y A. Puyana, La pobreza en América Latina - ¿Una dimensión olvidada de la integración económica regional?, 1a ed. ed., Buenos Aires: Consejo Latinoamericano de Ciencias Sociales - CLACSO, 2010, p. 143.

[4]Gobierno Autónomo Descentralizado Municipal de Isidro Ayora, «Gobierno Autónomo Descentralizado Municipal de Isidro Ayora. (14 de Mayo de 2019). Bienvenidosa Isidro Ayora,» 2019. [En línea]. Available: http://www.isidroayora.gob.ec/nh.html. [Último acceso: 2019].

[5]Programa de las Naciones Unidas para el Desarrollo, «OBJETIVOS DE DESARROLLO SOSTENIBLE,» 2019. [En línea]. Available: https://www.undp.org/ content/undp/es/home/sustainable-development-goals. html.

[6]H. Jaramillo Ordóñez y P. Jaramillo Luzuriaga, La justicia adminitrativa en el sistema oral, Primera ed., Loja: Offset Grafimundo, 2016, p. 323.

[7]M. Méndez Juez, «Transparencia pública en la regeneración democrática: La necesidad de visibilizar las agendas institucionales en España,» Cuadernos de Gobierno y Administración Pública, vol. 4, nº 1, pp. 89-
$105,2017$.

[8]J. M. Velázquez Gutiérrez, «Retroalimentando la etapa de terminación: Análisis de casos de políticas públicas,» Estudios Políticos, novena época, $n^{\circ} 38$, pp. 163-189, mayo-agosto 2016.

[9]V. D. García García, «Evaluación de políticas públicas juveniles en tres municipios del Estado de México (2013-2015),» Revista Latinoamericana de Ciencias Sociales, Niñez y Juventud, vol. 15, no 1, pp. 403-418, 2017.

[10]Centro Latinoamericano de Administración para el Desarrollo, «Gestión Pública Iberoamericana para el siglo XXI,» XL Reunión Ordinaria del Consejo Directivo del CLAD, pp. 1-23, 2010.

[11]A. Caso Raphael, «Gestión y presupuesto para resultados en el contexto de la Nueva Gestión Pública (NGP) en México,» de La evaluación de políticas públicas en México, México, D.F., Géminis Editores e impresores, S.A. de C.V, 2011, p. 162.

[12]J. G. Rodríguez y A. Sánchez Riofrío, «TIC y pobreza en América Latina,» Iconos. Revista de Ciencias Sociales, $n^{\circ}$ 57, p. 21, 2017.

[13]M. C. Calderón, «Normas sociales y umbrales de la pobreza,» Acta sociológica, no 70, pp. 73-98, mayo-agosto 2016.

[14]Secretaría Nacional de Planificación y Desarro1lo, «Plan Nacional de Desarrollo 2017-2021-Toda una Vida,» 22 Septiembre 2017. [En línea]. Available: http://www.planificacion.gob.ec/wp-content/uploads/ downloads/2017/10/PNBV-26-OCT-FINAL_0K.compressed1.pdf.

[15]C. I. Becerril Velasco, «El papel del Estado en el alivio a la pobreza en la era neoliberal Una aproximación teórica,» $\mathrm{n}^{\circ}$ 225, p. Revista Mexicana de Ciencias Políticas y Sociales, Septiembre 2015.

[16]A. Alonso Serrano, L. García Sanz, I. León Rodrigo, E. García Gordo, B. Gil Álvaro y L. Rios Brea, «Métodos de investigación de enfoque experimental,» pp. 167-193, 2012.

[17]A. Eslava, «Análisis cualitativo y cuantitativo para los estudios políticos. Trilateralismo, metodología experimental y Grounded,» Cinta moebio, $n^{\circ}$ 51, pp. 111126, 2014. 